\title{
Should all adult cystic fibrosis patients with repeated nontuberculous mycobacteria cultures receive specific treatment? A 10-year case-control study
}

\author{
To the Editor: \\ The disease caused by nontuberculous mycobacteria (NTM) forms a broad clinical spectrum from \\ asymptomatic infection to fatal disseminated disease in humans. While many NTM patients have \\ underlying pulmonary diseases, NTM symptoms are undifferentiated from symptoms of patients with \\ chronic pulmonary structural diseases [1].
}

The 2007 American Thoracic Society (ATS)/Infectious Diseases Society of America (IDSA) recommendations on NTM pulmonary diseases define an obligatory treatable pulmonary infection with NTM by the presence of all the following criteria: clinical (i.e. respiratory symptoms), radiological (i.e. nodular or cavitary opacities on chest radiograph, or multifocal bronchiectasis with multiple small nodules on high-resolution computed tomography) and microbiological criteria (detection of NTM by culture in respiratory samples, at least two positive sputum cultures or one positive bronchoalveolar lavage fluid culture) [2]. Many cystic fibrosis (CF) patients already fulfil these clinical and radiological criteria due to the underlying disease. Therefore, the clinical interpretation of repeated NTM detection in the sputum of CF patients is difficult. The guidelines state that "The diagnostic criteria and treatment regimens for NTM pulmonary infection in patients with CF are the same as for patients without $\mathrm{CF}$, although they may be more difficult to apply because of underlying disease and concomitant infections (C, III)." [2].

We aimed to assess the impact of repeated NTM detection fulfilling the microbiological ATS/IDSA criteria on the long-term course of patients with CF. Before the aforementioned guidelines were implemented in our institution (Dept of Pulmonary Medicine, Hannover Medical School, Hannover, Germany), NTM treatment was restricted to selected patients with Mycobacterium abscessus only. The decision about treatment initiation in $M$. abscessus patients was nonstandardised and at the discretion of the treating physician. Therefore, we had the chance to observe the clinical course of untreated CF patients with repeated detection of NTM over a long observational period.

We performed a single centre chart review of all 186 adult CF patients treated at the adult CF clinic of the Hannover Medical School (Hannover, Germany). At our clinic, patients are seen at least four times per year and sputum samples are obtained at each visit. We identified 26 (14\%) patients with positive microbiological criteria for NTM infection according to the ATS/IDSA guidelines. These 26 patients were matched with $46 \mathrm{CF}$ patients without NTM detection. The following matching criteria with values at the time of first NTM detection were applied: age ( \pm 5 years), sex, forced expiratory volume in $1 \mathrm{~s}(\mathrm{FEV} 1)$ ( $\pm 15 \%$, best value in the year of first NTM detection or matching, respectively) and body mass index (BMI) $\left( \pm 2 \mathrm{~kg} \cdot \mathrm{m}^{-2}\right.$, best value in the year of first NTM detection or matching, respectively). At the time of NTM detection or matching, chest radiography results were available for 21 NTM patients and 38 controls. All chest radiographs showed proof of bronchiectasis except in two NTM patients and two controls.

Characteristics of the study population at the time of matching for NTM patients and controls were as follows: age: 25.3 years and 24.7 years, respectively; BMI $19.8 \mathrm{~kg} \cdot \mathrm{m}^{-2}$ and $19.8 \mathrm{~kg} \cdot \mathrm{m}^{-2}$, respectively; FEV1 $58.4 \%$ and $59.3 \%$, respectively; and proportion of females: $34.6 \%$ and $32.6 \%$, respectively.

We compared the clinical course over a period of 10 years ( 5 years before and 5 years after first detection of NTM). We started the observation 5 years before the first detection of NTM to rule out that NTM detection was associated randomly with a possible faster deterioration in individual patients.

T-test for heterogeneous variances (Welch test) and for nominal scaled data, the Chi-squared test and the exact Fisher test were used as inference statistical methods for steady data.

The following mycobacterial species were detected repeatedly in the 26 patients with NTM: Mycobacterium avium intracellulare complex (MAC) $(\mathrm{n}=14)$, M. abscessus $(\mathrm{n}=10)$, and Mycobacterium gordonae $(\mathrm{n}=2)$. Only five out of 10 patients with $M$. abscessus and one patient with MAC received treatment according to ATS/IDSA recommendations.

Infections with other pathogens at the time of NTM detection or matching revealed no significant differences for Pseudomonas aeruginosa, Aspergillus fumigatus, Burkholderia cepacia, Stenotrophomonas maltophilia, 
Staphylococcus aureus, Haemophilus infuenzae and Enterobacteriacae. Methicillin-resistant S. aureus was more frequently detected in the NTM group (six out of 26 versus two out of 46, $\mathrm{p}=0.023$ ). The proportion of patients receiving long-term azithromycin treatment was similar ( 16 out of 26 versus 37 out of 46, $\mathrm{p}=0.081$ ).

Seven patients underwent lung transplantation (controls: $\mathrm{n}=3$; patients with MAC: $\mathrm{n}=2$; patients with $M$. abscessus: $\mathrm{n}=2$ ). Only one of these patients received antimycobacterial treatment for $M$. abscessus before transplantation. The frequency of exacerbation, CF-associated diseases and the requirement of additional supportive measures were distributed equally in both groups.

During the observation period the median FEV1 of the NTM patients declined by $9.5 \%$ (range $-39-13 \%$ ) and by $19.4 \%$ in the matched controls (range $-56--2 \%, \mathrm{p}=0.066$ ).

The same was observed for median (range) $\Delta$ vital capacity (NTM: $-3.5 \%$ ( $-26-14 \%$ ); controls: $-16.0 \%$ $(-40-10 \%), \mathrm{p}=0.526)$, arterial oxygen tension (NTM: $-8 \mathrm{mmHg}(-16-10 \% \mathrm{mmHg})$; controls: $-8 \mathrm{mmHg}$ $(-25--1 \mathrm{mmHg}), \mathrm{p}=0.095)$ and arterial carbon dioxide tension (NTM: $1.5 \mathrm{mmHg}(-4-12 \% \mathrm{mmHg}$; controls: $2 \mathrm{mmHg}(-5-14 \% \mathrm{mmHg}), \mathrm{p}=0.619)$. These intergroup differences were nonsignificant. In the NTM group, there was no accelerated decline in lung function after NTM detection (figure 1). Exacerbation rate did not differ between cases and controls $(\mathrm{p}=0.972)$. Patients were ranked according to the number of exacerbations within the 5 years after matching/first NTM detection. The proportion of NTM patients and controls within the individual groups was distributed equally: 0-3 exacerbations: $50 \%$ and $50 \%$, respectively; $4-7$ exacerbations: $34.6 \%$ and $37 \%$, respectively; $8-10$ exacerbations: $11.5 \%$ and $8.7 \%$, respectively; and $>10$ exacerbations: $3.8 \%$ and $4.3 \%$, respectively.

Since the clinical relevance of $M$. gordonae is an issue of debate, we repeated the aforementioned analyses after exclusion of all patients infected with M. gordonae and all treated NTM patients. The trends were the same as above showing a more pronounced deterioration in the controls that reached no significant difference.

As in our study, a cohort study following 60 CF patients with NTM infections and 99 controls over 15 months found no significant effect on FEV1 [3]. Some studies in paediatric patients reported a faster decline in lung function in NTM infected patients $[4,5]$ or an improvement after antimycobacterial treatment [6]. In contrast to these studies, the patients observed in the study by OLIVIER et al. [3] and our study were all adults at the time of the first NTM detection: NTM $28.5 \pm 1.4$ years, controls $26.2 \pm 0.9$ years and NTM 25.3 \pm 6.6 years, controls $24.8 \pm 6.9$ years, respectively).

Furthermore, a recent Scandinavian study found that a higher proportion of patients who acquired NTM before the age of 20 years ended up developing end-stage lung disease (19\% versus 13\%, Ns) [7]. Taking these results together, an increased susceptibility to mycobacterial infections in younger patients cannot be excluded, which may be explained by immaturity of the cellular immunity [8]. However, all of the cited studies are small and limited in scope. The variability seen in results from study to study could also easily be explained by chance.

Assessing the impact of NTM on the clinical course of CF patients is difficult and requires large patient numbers and long observational periods. Whereas, to our knowledge so far, longest observation period is a

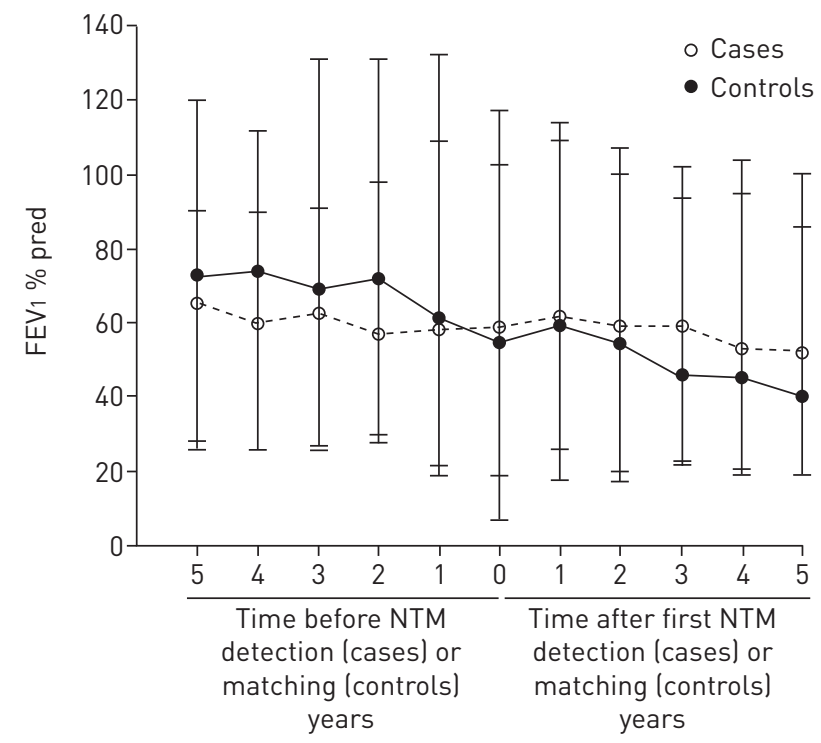

FIGURE 1 Course of forced expiratory volume in $1 \mathrm{~s}$ (FEV 1 ) in 26 cases and 46 controls 5 years before and 5 years after first detection of nontuberculous mycobacteria (NTM) or matching. 
strength, the limited patient number is a weakness of our study, even if some of the older studies enrolled even fewer patients [4-6]. In our study we did not find an impact on lung function decline; on the contrary, there was a trend towards a decelerated decline in lung function in patients with NTM that we currently cannot explain.

The indication for antimycobacterial combination therapy in CF patients with detected NTM is not straight-forward and has to balance the potential symptomatic improvement and uncertain prevention of progression against toxicity [9]. Furthermore, there may be advantages of early treatment of NTM in CF, including the unproven potential for improved eradication success and transplant implications of M. abscessus. However, even M. abscessus infection is no longer considered an absolute contraindication for lung transplantation [10].

According to our study and other studies, the ATS/IDSA criteria are more difficult to apply in CF patients and additional predictors for patients in need of NTM treatment would help to solve a common diagnostic dilemma. Recently suggested parameters include the number of repeated NTM detections, i.e. more than five compared to two to four [7], or the FEV1 decline in the prior year, i.e. $-5.8 \%$ per year versus $-0.7 \%$ per year [11].

@ERSpublications

Not all CF patients with MAC detected in respiratory samples require anti-mycobacterial treatment http://ow.ly/X66gt

Christiane Albrecht ${ }^{1}$, Felix Ringshausen ${ }^{1}$, Sebastian Ott $^{2}$, Dirk Wagner ${ }^{3}$, Jessica Rademacher ${ }^{1}$, Michael Schneider ${ }^{4}$, Tobias Welte ${ }^{1}$ and Mathias W. Pletz ${ }^{5}$

${ }^{1}$ Dept of Pulmonary Medicine, Hannover Medical School, German Center for Lung Research (DZL), Hannover, Germany. ${ }^{2}$ Dept of Pulmonary Medicine, University Hospital Bern (Inselspital) and University of Bern, Bern, Switzerland. ${ }^{3}$ Dept of Infectious Diseases and Center for Chronic Immunodeficiency, University Medical Center, Freiburg, Germany. ${ }^{4}$ Center for Biometry, Medical Informatics and Medical Technology, Hannover Medical School, Hannover, Germany. ${ }^{5}$ Center for Infectious Diseases and Infection Control, Jena University Hospital, Jena, Germany.

Correspondence: Mathias W. Pletz, Center for Infectious Diseases and Infection Control, Jena University Hospital, Erlanger Allee 101, 07740 Jena, Germany. E-mail: mathias.pletz@med.uni-jena.de

Received: July 282015 | Accepted after revision: Dec 232015 | First published online: Feb 042016

Support statement: M.W. Pletz was supported by a grant from the German Federal Ministry of Education and Research (BMBF); grant numbers 01KI1204. Funding information for this article has been deposited with FundRef

Conflict of interest: Disclosures can be found alongside the online version of this article at erj.ersjournals.com

\section{References}

1 Mirsaeidi M, Farnia P, Sadikot R, et al. Nontuberculous mycobacteria: epidemiologic, mycobacteriologic, and clinical aspects. Biomed Res Int 2015; 2015: 523697.

2 Griffith DE, Aksamit T, Brown-Elliott BA, et al. An official ATS/IDSA statement: diagnosis, treatment, and prevention of nontuberculous mycobacterial diseases. Am J Respir Crit Care Med 2007; 175: 367-416.

3 Olivier KN, Weber DJ, Lee JH, et al. Nontuberculous mycobacteria. II: nested-cohort study of impact on cystic fibrosis lung disease. Am J Respir Crit Care Med 2003; 167: 835-840.

4 Esther CR Jr, Esserman DA, Gilligan P, et al. Chronic Mycobacterium abscessus infection and lung function decline in cystic fibrosis. J Cyst Fibros 2010; 9: 117-123.

5 Esther CR Jr, Henry MM, Molina PL, et al. Nontuberculous mycobacterial infection in young children with cystic fibrosis. Pediatr Pulmonol 2005; 40: 39-44.

6 Forslow U, Geborek A, Hjelte L, et al. Early chemotherapy for non-tuberculous mycobacterial infections in patients with cystic fibrosis. Acta paediatrica 2003; 92: 910-915.

7 Qvist T, Gilljam M, Jonsson B, et al. Epidemiology of nontuberculous mycobacteria among patients with cystic fibrosis in Scandinavia. J Cyst Fibros 2015; 14: 46-52.

8 Lewinsohn DA, Gennaro ML, Scholvinck L, et al. Tuberculosis immunology in children: diagnostic and therapeutic challenges and opportunities. Int J Tuberc Lung Dis 2004; 8: 658-674.

9 Uyan ZS, Ersu R, Oktem S, et al. Mycobacterium abscessus infection in a cystic fibrosis patient: a difficult to treat infection. Int J Tuberc Lung Dis 2010; 14: 250-251.

10 Qvist T, Pressler T, Thomsen VO, et al. Nontuberculous mycobacterial disease is not a contraindication to lung transplantation in patients with cystic fibrosis: a retrospective analysis in a Danish patient population. Transplant Proc 2013; 45: 342-345.

11 Martiniano SL, Sontag MK, Daley CL, et al. Clinical significance of a first positive nontuberculous mycobacteria culture in cystic fibrosis. Ann Am Thorac Soc 2014; 11: 36-44. 